\title{
Monitoring intracellular, interstitial, and intravascular volume changes during fluid management procedures
}

\author{
Leslie D. Montgomery, Ph.D. ${ }^{1}$, Wayne A. Gerth, Ph.D. ${ }^{1}$, Richard W. Montgomery, Ph.D. ${ }^{1}$, \\ Susie Q. Lew, M.D. ${ }^{2}$, Michael M. Klein, M.D. ${ }^{3}$, Julian M. Stewart, M.D. ${ }^{3}$, and Manuel T. \\ Velasquez, M.D. ${ }^{2}$ \\ ${ }^{1}$ LDM Associates, San Jose, CA \\ ${ }^{2}$ George Washington University Medical Center, Washington, DC \\ ${ }^{3}$ New York Medical College, NY
}

\begin{abstract}
The bioimpedance spectroscopic (BIS) analytical algorithm described in this report allows for the non-invasive measurement of intravascular, interstitial, and intracellular volume changes during various fluid management procedures. The purpose of this study was to test clinical use feasibility and to demonstrate the validity of the BIS algorithm in computing compartmental volume shifts in human subjects undergoing fluid management treatment. Validation was performed using volume changes recorded from 20 end stage renal disease (ESRD) patients. The validation procedure involved mathematically deriving post hoc hematocrit profiles from the BIS data-generated fluid redistribution time profiles. These derived hematocrit profiles were then compared to serial hematocrit values measured simultaneously by a CritLine ${ }^{\circledR}$ monitor during 60 routine hemodialysis (HD) sessions. Regression and Bland Altman analyses confirm that the BIS algorithm can be used to reliably derive the continuous and real time rates of change of the compartmental fluid volumes. Regression results yielded a $\mathrm{R}^{2}>0.99$ between the two measures of hematocrit at different times during dialysis. The slopes of the regression equations at the different times were nearly identical, demonstrating an almost one to one correspondence between the BIS and CritLine ${ }^{\circledR}$ hematocrits. Bland Altman analyses show that the BIS algorithm can be used interchangeably with the CritLine ${ }^{\circledR}$ monitor for the measurement of hematocrit. The present study demonstrates for the first time that BIS can provide real-time continuous measurements of compartmental intravascular, interstitial and intracellular fluid volume changes during fluid management procedures when used in conjunction with this new algorithm.
\end{abstract}

\section{Introduction}

Hemodynamic redistribution of fluids between body segments and between the fluid compartments within those segments are of central importance to various fluid management procedures [4, 25] and disease states [20, 27, 28, 29, 30,34]. Such fluid redistributions affect cardiovascular function, water balance and perhaps skeletal muscle function through physiological mechanisms that may be better understood with simultaneous characterization of both the extent and the rate of the inter-compartment redistributions.

Please send correspondence to: Leslie D. Montgomery, 1764 Emory St., San Jose, CA 95126, 408-293-7447, pmontgomery@ telis.org. Conflict of Interest Statement

The authors from LDM Associates were the recipients of the National Heart, Lung and Blood Institute of the National Institutes of Health through SBIR Grants 1 R43 HL074524-01 and 2 R44 HL074524-02A2 entitled, "Intra/Extracellular Volume and Hemodynamics" between Sept. 1, 2003 and July 31, 2008. None of the authors have received or will receive any compensation or monetary benefit from the publication of this article. This article represents new research and has not been published elsewhere. 
Bioelectric impedance spectroscopic has proved useful for non-invasive monitoring of intercompartmental fluid shifts $[2,5,8,19,23,26,35,33,36$, among others.] To date, however, this approach has been limited to two-compartments: intra- and extra cellular volumes. The present paper demonstrates that, by using a new analytical algorithm, it is possible to derive the fluid shifts that take place between the intracellular, interstitial, and vascular compartments from the current BIS output data.

\section{Methods}

\subsection{Background}

Various resistance/capacitance (R/C) models $[1,6,21,24]$ are used to quantify segmental volumes from recorded impedance measurements. The $\mathrm{R} / \mathrm{C}$ model that is used by most currently available impedance systems represents the tissue as two parallel conductance paths: one through an extracellular compartment having average resistance (Re) and the other through an intracellular compartment having average resistance (Ri) and capacitance $(\mathrm{Cm})$. This model, which is limited to the determination of "intracellular" and "extracellular" resistances $\left(R_{i}\right.$ and $R_{e}$, respectively), can therefore only be used to estimate the corresponding intracellular, extracellular, and overall volumes of a monitored body segment. Unfortunately, an issue of principal concern in many clinical contexts is how the "extracellular volume" is distributed between its interstitial and intravascular compartments; an issue that the two component $\mathrm{R} / \mathrm{C}$ models cannot address.

Since both blood and bone are nearly wholly resistive over the frequency range used in current BIS instruments $[2,38]$ they should affect only the passage of current in the extracellular volume compartment. This configuration can be approximated by considering blood and bone to be additional fluid compartments that are each electrically in parallel with the intracellular and interstitial compartments of the other soft tissue, as illustrated in Figure 1 A below.

The complex admittance $\mathrm{Y}$ of the circuit shown in Figure 1A is given as a function of the applied current frequency $\mathrm{f}[17]$ by:

$$
Y=1 / Z=1 / \text { Rblood }+1 / \text { Rbone }+1 / R_{e}+1 / R_{i}+\left(1 / R_{i}\right) /\left[1+\left(R_{i} * C_{m} * j \omega\right) * *(1-\alpha)\right] .
$$

where:

$\mathrm{Z}$ is a series of complex impedances

$\mathrm{j}=$ square root of -1

$\omega=2 \pi f$ is the angular frequency

$\mathrm{R}_{\mathrm{e}}, \mathrm{R}_{\mathrm{i}}, \boldsymbol{a}$ and $\mathrm{C}_{\mathrm{m}}$ are obtained from resistance and reactance values recorded during each sweep of the frequency range used by the BIS.

An additional component of our analytical procedure is the development and application of the extravascular soft tissue cellular model $[16,17,32]$ illustrated in Figure 1B. This model can be used to calculate the interstitial and intravascular compartment volumes that comprise the previously calculated "extracellular" volume.

The extravascular soft tissue compartment of the monitored segment is modeled as a cylindrical homogeneous suspension of identical oblate spheroidal cells with long axes, a, oriented parallel to the applied field, I, as shown in Figure 1B. Cells are allowed to change volume only through variation in minor axis, $b$. The cell volume fraction and the intracellular and extracellular conductivities of this compartment are related to the compartment's Re, Ri, and Cm in Figure 1A with theory developed by Hugo Fricke [13-15]. 
The model shown in Figure 1B is based on an assumed physical structure of the monitored segment, particularly its extravascular compartment (Figure 1A), which governs the values and frequency dependence of the "resistive" and "reactive" components of the segmental impedance. The model is fit to each measured spectrum by an iterative numerical process in which values of the model parameters are found that bring model-prescribed spectra into closest possible agreement with the measured spectrum. Because the model is a function of more parameters than are uniquely determined by information in an impedance spectrum, selected model parameters must be assigned "fixed" values in the fitting process. Detailed descriptions of this analytical approach and the solution of Equation 1 can be found in Sasser et al., [32]; Gerth et al., [16]; and Gerth and Watke, [17].

\subsection{Validation}

The previous sections describe how swept frequency bioimpedance monitoring provides a convenient, minimally invasive means of monitoring intracellular, interstitial, and vascular volume changes. The question is whether these recordings reliably correspond to the actual volume changes in these three compartments. Absent invasive direct biochemical analysis of repeated fluid samples, it would be impossible to answer this question with absolute certainty. Fortuitously, however, HD treatment sessions provide ideal opportunities to monitor exogenously induced inter-compartmental fluid shifts with minimal additional procedures.

If the BIS net volume changes we compute do indeed provide a way to track three compartment fluid shifts, then such BIS-recorded fluid shifts should correlate with those of an independent physiologic measure of fluid volume changes. The HD patient's hematocrit (HCT), expressed as \%, rises (due to hemoconcentration) as excess fluids are removed by ultra-filtration (UF) - and falls or rises more slowly (due to hemodilution) as extravascular fluid moves into the vascular system as a result of compensatory osmotic and hydrodynamic pressure changes. Both changes in HCT and changes in the rate of vascular "refill" can be measured noninvasively during routine HD therapy sessions. Changes in HCT can be measured by a CritLine ${ }^{\circledR}$ optical monitor (HemaMetrics, Kaysville, UT). The rate of "refill" can be calculated from the BIS volume data.

\subsection{Subject population}

Data used to validate our analytical procedures were collected from a series of dialysis treatments conducted at the New York Medical Center (NYMC). The validation protocol was fully approved by the appropriate Internal Review Boards prior to study initiation. Twenty chronic HD patients over age 21 who were capable of giving informed consent underwent testing. Each patient was tested during a nephrologist prescribed treatment using Polyflux Series (8L, 170H or 210H) high flux dialyzers (Hechingen, Germany) for 3 to 4 hours, and was tested three times on subsequent days during a given week. In this way, a total of 60 tests were conducted at NYMC. All testing was conducted in a temperature controlled room $\left(24-26^{\circ} \mathrm{C}\right)$.

Eleven male and nine female patients were included in the test group. Even though both male and female patients were tested, gender response differences were not the focus of this work. Females, who have lower hematocrits [22] were included to provide a wider range of hematocrit values.

The mean age of the 20 subjects was $65.3 \pm 11.3$ (yrs). Their Pre and Post dialysis HCT (\%) were $34.84 \pm 2.50$ and $38.47 \pm 3.60$, respectively. Their Pre and Post dialysis weights $(\mathrm{Kg})$ were $78.78 \pm 16.17$ and $76.53 \pm 15.91$, respectively. The HCT and weight changes that took 
place during dialysis were $3.63 \pm 1.90(\%)$ and $2.25 \pm 1.13(\mathrm{Kg})$, respectively. A total mean of $2413 \pm 776.2(\mathrm{ml})$ of fluid was removed during the 60 dialysis treatments.

\subsection{Instrumentation}

A tetra-polar BIS (UFI Inc., Morro Bay, CA) was used to measure the tissue resistance and reactance at 40 discrete frequencies once a minute during this study. The BIS electrodes were attached to the subject's dominant lower leg as shown in Figure 2.

The source electrodes were placed just above the knee and just above the lateral malleolus; the sampling electrodes were placed just below the knee and above the ankle. After a 30minute semi-reclined acclimatization/instrumentation period, the measurements listed above were recorded for a period of 30 minutes prior to HD. These measurements served as the control values for the intra-HD and post-HD recovery period values. Systolic and diastolic blood pressure (brachial cuff measures) and heart rate were measured every 30 minutes during the dialysis procedure as per dialysis facility protocol. Body weights were measured before and after each HD session. Possible signs and symptoms of hypovolemia such as breathlessness, dizziness, presyncope, nausea, vomiting, thirst, fatigue, and cramps were monitored and recorded by the attending medical officer. Mid -session dialysis prescription alterations such as changes in dialysate and blood flow rates and net fluid removal were recorded continuously by the dialysis machine.

A CritLine ${ }^{\circledR}$ optical monitor served as the "gold standard" for validation and was used simultaneously to detect on-line changes in hematocrit. The CritLine ${ }^{\circledR}$ measures the optical absorption and scattering properties of red blood cells as they pass through the HD circuit. Since red blood cell mass and hemoglobin do not usually change during treatment, the relative changes in hematocrit should parallel the changes in blood fluid content. The CritLine ${ }^{\circledR}$ instrument is well

\subsection{Data analysis}

Three calf compartment and total calf compartment net fluid volume changes are displayed in real-time by the BIS monitor we have developed. This is shown as Figure 3.

The recorded minute-to-minute changes in each of these time series are used to calculate fluid shifts across compartments. The calculation takes advantage of the fact that intracellular fluid can only move into (or come from) the interstitial compartment; there is no other adjacent compartment. This implies that any change in intracellular fluid volume $(\mathrm{Vc})$ must reflect a movement of fluid between the intracellular and interstitial compartments. Thus, using the notation "Mic" for fluid "movement" into cells from the interstitial compartment, (ML/minute):

$$
\mathrm{Mic}=\Delta \mathrm{Vc} \quad(2)
$$

The sign of Mic indicates the direction of movement. A negative value indicates an outflow from the intracellular compartment in to the interstitial compartment.

Proceeding in this manner, the movement of fluid from the vascular (blood) compartment (Mbi) into the interstitial compartment and the movement of fluid from other segments (Mob) into the vascular compartment are calculated via a block triangular linear transformation of the compartment volume changes:

$$
\mathrm{Mbi}=\Delta \mathrm{Vc}+\Delta \mathrm{Vi}
$$


Recalling that fluid movement (ML/min) is here defined as movement into the indicated compartment, a negative value of Mbi is a net movement of interstitial (or extravascular) fluid into the vascular compartment. Hereafter this will be referred to as "vascular refill," and such refill will be assumed to offset some of the effect of ultrafiltration (UF) on hematocrit. That is, as UF tends to raise the value of HCT, a negative value of Mi tends to decrease HCT via hemodilution.

This relationship derived from the BIS data is then compared with minute-by-minute changes in HCT as recorded independently by the CritLine ${ }^{\circledR}$ optical device. These two variables are hereafter labeled $\triangle$ REFILL and $\triangle$ CLHCT, and the comparison consists of a simple least-squares regression: $\triangle$ CLHCT $=\alpha+\beta$ ( $\triangle$ REFILL), where $\alpha$ is the $Y$ axis intercept and $\beta$ is the slope coefficient of the regression equation.

\subsection{Statistics}

Statistical analysis was performed using MedCalc Ver. 10.0.0.0. A Student t test for paired observations was used to compare pre- and post-hemodialysis results. Tests of significant differences between the BISHCT and CLHCT values at the specified times were performed by non-paired Student $t$ test. Additional Pearson correlation and Bland-Altman (Bland Altman, 1996) analyses were used to compare the BISHCT and CLHCT results at each time. Results are reported as mean $\pm \mathrm{SD}$. The variance in the results given for the analyses described in Section 3.1 and listed in Table 1 are reported as the SE of the estimate.

First-difference analyses were used in the calculation of BISHCT values from the impedance data to mitigate concern about spurious correlation due to autocorrelation in 'undifferenced' time series. Durbin-Watson [7] and Augmented Dickey-Fuller [31] tests were employed nonetheless to assure that first-differenced series were not autocorrelated and were stationary but still cointegrated. Statistical tests also confirmed that $\triangle$ REFILL "Granger-causes" [18] $\Delta$ CLHCT.

\section{Results}

\subsection{Controlled sample results}

Of the 60 sessions completed, 12 were best suited for validation procedures as they involved no changes in the UF-rate, no patient complications, and no interventions (such as therapeutic infusions). Table 2 presents the regression results for this subset of HD sessions. The 'observations' indicated are the number of minutes of elapsed time during which the regression data were recorded.

The t-values for the estimate of the slope coefficient $(\beta)$ indicate that the null-hypothesis of no relationship between $\triangle$ REFILL and $\triangle$ CLHCT can safely be rejected ( $\mathrm{p} \ll 0.01$ in every case). The standard errors (SE) are very small, ranging .005057-.021884. For example, assuming that nominal hematocrit values are approximately 36 during dialysis, $\mathrm{SE}=$ 0.00507 (as in the first session above) implies that $99 \%$ (3 SD) of any errors in "predicting" changes in CLHCT from the BIS data will be within $36+/-.01521$ (i.e. within four thousandths of one percent of the HCT value).

The remarkably close relationship between the changes in "vascular refill" calculated from the BIS data and changes in CLHCT implies that the (un-differenced) trajectories of these two variables will be very close. This can easily be illustrated by time-integrating both 
series, and setting the constant of integration equal to the "actual" HCT value as an initial condition. This is illustrated in Figure 4 using the estimated coefficients for the first session shown in Table 1.

This same procedure was used to produce graphs like Figure 4 for all 60 HD sessions. The strength of correlation between the two series for all sessions was evaluated by "sampling" all of the charts at a specified elapsed time, thereby obtaining 60 pairs of values (i.e., of CLHCT and its predicted value). This sampling procedure was repeated for several intervals selected on the basis of the following considerations.

All dialysis sessions included in this analysis lasted at least 120 minutes. Shortly after 120 minutes some of the dialysis sessions were terminated for different reasons; patients experiencing syncope, cramps, completion of the required dialysis, etc. Mean calculated BIS and observed CritLine ${ }^{\circledR}$ hematocrit $( \pm \mathrm{SD}$ ) values are presented in Table 2 for elapsed dialysis times of 30,60, 90, 120, and END. The END values that are given in Table 2 were taken from the last minute before cessation of dialysis. The average $( \pm \mathrm{SD})$ time for the END data is $183.4 \pm 13.1 \mathrm{~min}$. from the start of dialysis. The CritLine ${ }^{\circledR}$ hematocrit values measured by the optical sensor in Table 3 and the following tables and figures are designated CLHCT. The values calculated from the BIS measurements are designated by BISHCT. No significant differences were found between the BISHCT and the CLHCT values at any of the elapsed times listed in Table 2.

Regression and Bland Altman [3] analyses were performed to compare the BIS hematocrit values to the observed CritLine ${ }^{\circledR}$ values at each of the elapsed times listed in Table 3 Graphical results of the regression and Bland Altman analyses at the specified elapsed times are given in Figure 5. Regression charts of BISHCT vs. CLHCT at elapsed times of 30, 60, 90, 120 min and during the last minute of dialysis are given in Figures 5 - A, C, E, G, and I, respectively. The solid trace in each of these charts represents the corresponding regression equation and the dashed traces show the $95 \%$ confidence limits of the regression. The regression equations and $\mathrm{R}^{2}$ values for each of these comparisons are given in the appropriate panels within Figure 5.

The differences between BISHCT and CLHCT are represented in Bland Altman graphs [3] for the same elapsed times in Figures 5 - B, D, F, H, and J, respectively. The mean difference at each time is shown by the solid horizontal line, the limits of agreement is shown by the large dashed traces, and the $95 \%$ confidence interval is illustrated by the small dashed lines in Figures 5 -B, D, F, H, and J. Table 3 lists the corresponding Bland Altman results.

\section{Discussion}

This study describes how a new algorithm can be applied to derive the intracellular, interstitial, and intravascular fluid volume changes from the data provided by current BIS instruments. Intra-dialysis fluid compartmental volume changes were measured by a CritLine ${ }^{\circledR}$ and these measurements were used to demonstrate and confirm the BIS algorithm results.

The results presented in Table 1 represent the subset of treatments during which patients required no intervention for hemodynamic change. These results demonstrate that a simple linear model can be used to compare the BISHCT values derived from the intracellular, interstitial, and intravascular compartmental volume changes to the CLHCT measurements.

Regression and Bland Altman analyses were then performed using the data recorded during treatments of the entire cohort ( 60 dialysis sessions) to determine if the linear model is 
applicable to dialysis sessions during which patients require clinical intervention such as changes to ultrafiltration or flow rates. This data included results from dialysis sessions with varying UF rates and changes in patient posture, and sessions during which fluid or medications were administered or food consumed.

The $\mathrm{R}^{2}$ values obtained from the regression analyses shown in Figure 5 are all greater than 0.9 , ranging from 0.993 to 0.994 , thus demonstrating a very close relationship between BISHCT and CLHCT. In addition, the slope coefficients of the various regression equations given in Table 3, are all nearly identical confirming that there is almost a one to one correspondence between BISHCT and CLHCT at each of the elapsed times

The Bland Altman analysis (Table 3) suggests that there are no significant differences between the BISHCT and CLHCT values at any of the elapsed times. The mean difference between BISHCT and CLHCT was 0.06066 at the end of dialysis. This does indicate a slight over-estimation of the BISHCT as the time of treatment continues. However, this difference is negligible when the two actual hematocrit values are compared. Given the mean hematocrit of 38.22 for the CLHCT at the end of dialysis from Table 2 and the corresponding mean difference for that time from Table 3 of 0.06066 , the actual error is $0.15 \%$ of the CLHCT.

The ability to differentiate between volume changes in the three fluid compartments during fluid management therapy will fill a critical need in many health care situations. For example, in critically ill patients who are hypotensive, such as in septic shock, ICU physicians often give large amounts of fluids (as much as 10 or more liters a day) to maintain blood pressure and hemodynamic stability. Ideally, the replacement fluid should stay intravascular to raise blood pressure. Unfortunately, if this fluid moves into the interstitial space, this will lead to massive edema and if it goes into lung tissue, it leads to acute respiratory distress syndrome (ARDS), which is associated with high mortality. Treatment of this complication requires fluid removal by diuretic therapy in patients with normal kidney function and renal replacement procedures in those with kidney failure. Usually these patients require invasive monitoring such as intra-arterial catheters to measure $\mathrm{BP}$, and placement of central venous catheters to measure wedge pressures, pulmonary arterial pressure, and cardiac output. Continuous calculation and display of the intracellular, interstitial and intravascular volume changes would provide a better guide in fluid management in such patients, so that fluid administration could be adjusted only after intravascular repletion was obtained and before volume-overload occurred.

Our BIS analytical procedure will also be useful for intravenous fluid management in other acute settings such as patients suffering from septic or cardiogenic shock, extensive burns, lymphedema, or perhaps critical patients in Emergency or Operating Rooms. Our noninvasive procedure allows bedside monitoring of fluid shifts and, in doing so, informs individualized, immediate clinical decision-making regarding fluid resuscitation.

The general algorithm described in this work may be used in conjunction with the output data from other BIS instruments. However, most of our work to date has been in applications to data from the human calf, and we expect that its use in application to whole legs or arms will require some modifications to better represent the cells in those body segments.

In addition, specification of appropriate parameter values and of the initial conditions used in the application of the algorithm to calculate interstitial volumes may be different for different body segments that cannot be approximated as a cylinder or contain nonconductive voids, such as the head and torso. Each body segment may require a different set of cellular parameters for a given application. 
The present study demonstrates that a new BIS algorithm can be used to provide real-time continuous measurements of intracellular, interstitial, and intravascular fluid volume changes during fluid management procedures. Such information may prove valuable in the diagnosis and management of rapid changes in body fluid balance.

\section{Acknowledgments}

This work was funded, in part, by the National Heart, Lung and Blood Institute of the National Institutes of Health through SBIR Grants 1 R43 HL074524-01 and 2 R44 HL074524-02A2 entitled, "Intra/Extracellular Volume and Hemodynamics" between Sept. 1, 2003 and July 31, 2008. The authors wish to thank Ms. Sharon Hanish, Brian Scholfield, and Marty Loughry of UFI, Inc., Morro Bay, CA for their technical support and encouragement during this project. We especially want to thank Dr. Jennifer E. Flythe of Brigham and Women's Hospital, Harvard Medical School for her assistance in editing this manuscript.

\section{References}

1. Ackman JJ, Seitz MA. Methods of complex impedance measurement in biologic tissue. CRC Critical Reviews in Biomedical Engineering. 1984; 11:281-311.

2. Bartok C, Schoeller DA. Estimation of segmental muscle volume by bioelectrical impedance spectroscopy. J Appl Physiol. 2004; 96(1):161-6. [PubMed: 14506096]

3. Bland J, Altman DG. Statistical methods for assessing agreement between two methods of clinical measurement. Lancet. 1996; 1:307-313. [PubMed: 2868172]

4. Boyd JH, Forbes J, Nakada T, Walley KR, Russell JA. Fluid resuscitation in septic shock: A positive fluid balance and elevated central venous pressure are associated with increased mortality. Crit Care Med. 2011; 39(2):259-65. [PubMed: 20975548]

5. Chertow GM, Lowrie EG, Wilmore DW, Gonzalez J, Lew NL, Ling J, Leboff MS, Gottlieb MN, Huang W, Zebrowski B. Nutritional assessment with bioelectrical impedance analysis in maintenance HD patients. J Am Soc Nephrol. 1995; 6(1):75-81. [PubMed: 7579073]

6. Cole KS, Cole RH. Dispersion and absorption in dielectrics. J Chem Phys. 1941; 9:341-352.

7. Durbin J, Watson GS. Testing for serial correlation in least squares regression, I. Biometrika. 1950; 37:409-428. [PubMed: 14801065]

8. Ellis KJ, Wong WW. Human hydrometry: Comparison of multifrequency bioelectrical impedance with 2H2O and bromine dilution. J Appl Physiol. 1998; 85(3):1056-1062. [PubMed: 9729583]

9. Federal Drug Administration. K011741 Premarket Approval Crit-Line IIITQA URR Monitor. 2002

10. Federal Drug Administration . K093834 Premarket Approval Crit-Line Anemia Management Monitor. 2010

11. Fricke H. A mathematical treatment of electric conductivity and capacity of disperse systems. I. The electric conductivity of a suspension of homogeneous spheroids. Phys Rev. 1924; 24:575587.

12. Fricke H. A mathematical treatment of electric conductivity and capacity of disperse systems. II. The capacity of a suspension of conducting spheroids surrounded by a nonconducting membrane for a current of low frequency. Phys Rev. 1925; 26:678-681.

13. Fricke H. The electric capacity of suspensions of red corpuscles of a dog. Phys Rev. 1925; 26:682687.

14. Fricke H, Morse $\mathrm{S}$. The electric resistance and capacity of blood for frequencies between 800 and 4.5 million cycles. J Gen Physiol. 1925; 9:153-167. [PubMed: 19872239]

15. Fricke $H$. The electric permittivity of a dilute suspension of membrane-covered ellipsoids. J Appl Phys. 1953; 24:644-646.

16. Gerth, WA.; Montgomery, LD.; Wu, YC. Proceedings of Third Annual IEEE Symposium on Computer-Based Medical Systems. IEEE Computer Society Press; Chapel Hill, NC: 1990. A computer-based bioelectrical impedance spectroscopic system for noninvasive assessment of compartmental fluid redistribution; p. 446-453.

17. Gerth WA, Watke CM. Electrical impedance spectroscopic monitoring of body compartment volume changes. J Clin Eng. 1993; 18(3):253-260. 
18. Granger CWJ. Investigating causal relations by econometric models and cross-spectral methods. Econometrica. 1969; 37 (3):424-438.

19. Hannan WJ, Cowen SJ, Plester CE, Fearon KCH, DeBeau A. Comparison of bio-impedance spectroscopy and multi-frequency bio-impedance analysis for the assessment of extracellular and total body water in surgical patients. Clin Science. 1995; 89:651-658.

20. Jaeger JQ, Mehta RL. Assessment of dry weight in hemodialysis: An overview. JASN. 1999; 10:392-403. [PubMed: 10215341]

21. Jindal GD. Impedance plethysmography for screening vascular disorders. J Postgrad Med. 1986; 32:1-3. [PubMed: 3746705]

22. Kameneva MV, Watach MJ, Borovetz HS. Gender difference in rheologic properties of blood and risk of cardiovascular diseases. Clinical Hemorheology and Microcirculation. 1999; 21:357-363. [PubMed: 10711771]

23. Kamimura MA, Dos Santos NSJ, Avesani CM, Canziani MEF, Draibe SA, Cuppari L. Comparison of three methods for the determination of body fat in patients on long-term hemodialysis therapy. $\mathrm{J}$ Am Diet Assoc. 2003; 103:195-199. [PubMed: 12589325]

24. Kanai H, Katsuyuki S, Haeno M. Electrical measurement of fluid distribution in human legs: Estimation of extra- and intracellular fluid volume. J Microwave Power. 1983; 18:233-43.

25. Maisch B, Dristic A. Practical aspects of the management of pericardial disease. Heart. 2003; 89:1096-1103. [PubMed: 12923044]

26. Mendley SR, Majkowski NL, Schoeller DA. Validation of estimates of total body water in pediatric dialysis patients by deuterium dilution. Kidney Int. 2005; 67:2056-2062. [PubMed: 15840057]

27. Montgomery LD, Dietrich MS, Armer JM, Stewart BR, Ridner SH. Segmental blood flow and hemodynamic state of lymphedematous and non-lymphedematous arms. Lymphatic Research and Biology. 2011; 9(1):31-42. [PubMed: 21417765]

28. Montgomery LD, Hanish HM, Marker RA. An impedance device for study of multisegmental hemodynamic changes during orthostatic stress. Aviat Space Environ Med. 1989; 60:116-22.

29. Nanovic L. Electrolytes and fluid management in hemodialysis and peritoneal dialysis. Nutr Clin Pract. 2005; 20(2):192-201. [PubMed: 16207656]

30. Ridner SH, Montgomery LD, Hepworth JT, Stewart BR, Armer JM. Comparison of upper limb volume measurement techniques and arm symptoms between healthy volunteers and individuals with known lymphedema. Lymphology. 2007; 40(1):35-46. [PubMed: 17539463]

31. Said SE, Dickey DA. Testing for unit roots in autoregressive-moving average models of unknown order. Biometrika. 1984; 71:599-607.

32. Sasser DC, Gerth WA, Wu YC. Monitoring of segmental intra- and extracellular volume changes using electrical impedance spectroscopy. J Appl Physiol. 1993; 74:2180-2187. [PubMed: 8335546]

33. Shulman T, Heidenheim AP, Kianfar C, Shulman SM, Lindsay RM. Preserving central blood volume: Changes in body fluid compartments during hemodialysis. ASAIO J. 2001; 47:615-618. [PubMed: 11730198]

34. Stewart JM, Medow MS, Glover JL, Montgomery LD. Persistent splanchnic hyperemia during upright tilt in postural tachycardia syndrome. Am J Physiol Heart Circ Physiol. 2006; 290:H665H673. [PubMed: 16143646]

35. Van Den Ham ECH, Kooman JP, Christiaans MHL, Nieman FHM, Van Kreel BK, Heidendal GAK, Van Hooff JP. Body composition in renal transplant patients: Bioimpedance analysis compared to isotope dilution, dual energy X-ray absorptiometry, and anthropometry. J Am Soc Nephrol. 1999; 10:1067-1079. [PubMed: 10232694]

36. Van Marken Lichtenbelt WD, Snel YEM, Brummer RJM, Koppeschaar HPF. Deuterium and bromide dilution, and bioimpedance spectrometry independently show that growth hormonedeficient adults have an enlarged extracellular water compartment related to intracellular water. $\mathbf{J}$ Clin Endro Met. 2005; 82(3):907-911.

37. van der Sande FM, Kooman JP, Barendregt JNM, Nieman FHM, Leunissen KML. Effect of intravenous saline, albumin, or hydroxyethylstarch on blood volume during combined ultrafiltration and hemodialysis. J Am Soc Nephrol. 1999; 10:1303-1308. [PubMed: 10361869] 
38. van der Sande FM, Luik AJ, Kooman JP, Verstappen V, Leunissen KML. Effect of intravenous fluids on blood pressure course during hemodialysis in hypotensive-prone patients. J Am Soc Nephrol. 2000; 11:550-555. [PubMed: 10703679] 
Fig 1A

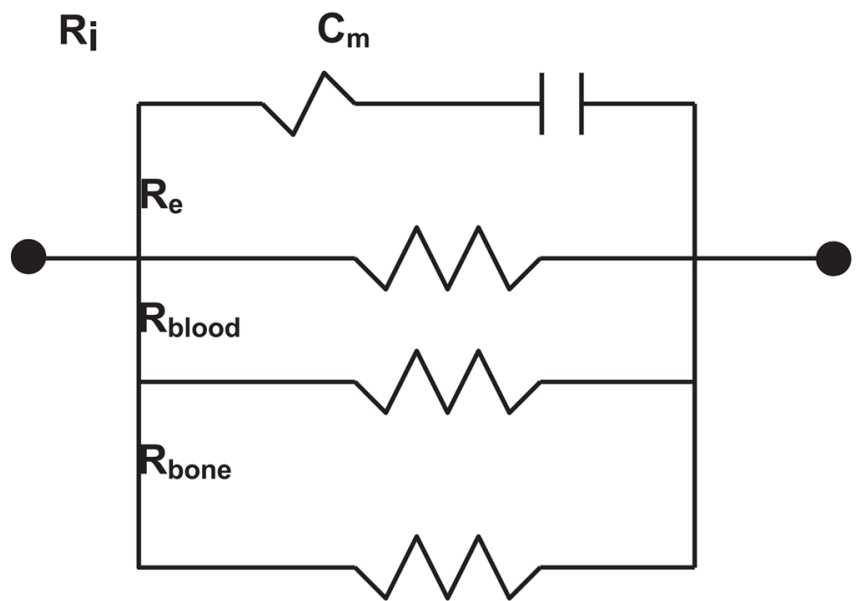

Fig 1B

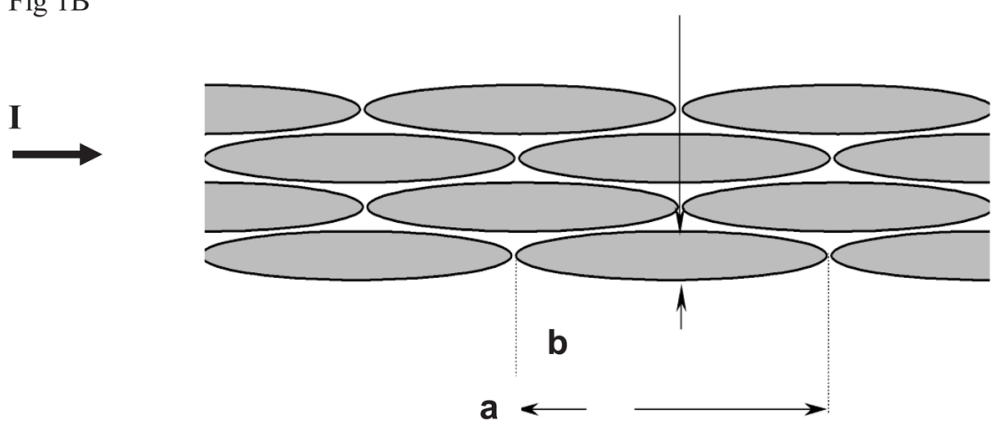

Figure 1.

Figure 1.A Equivalent circuit diagram used in BIS analysis

Figure 1.B Extra-vascular soft tissue compartment model used in calculation of intravascular and interstitial volumes 


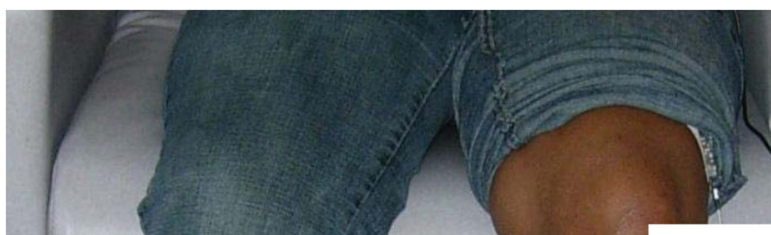

Figure 2.

BIS electrode placement 


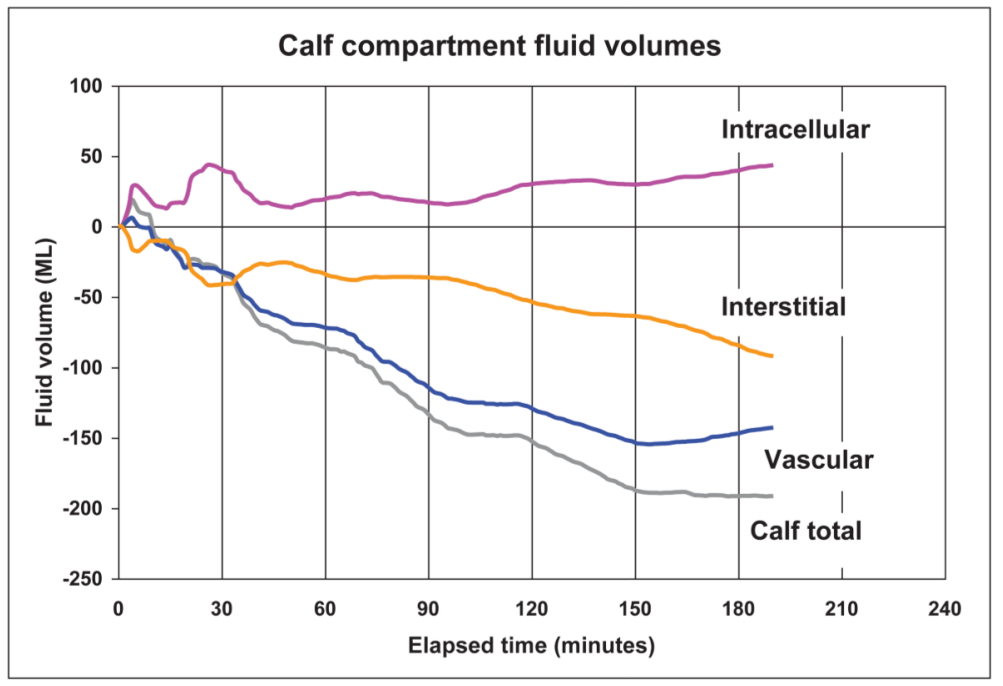

Figure 3.

Kalman-smoothed fluid compartment volumes relative to initial values 


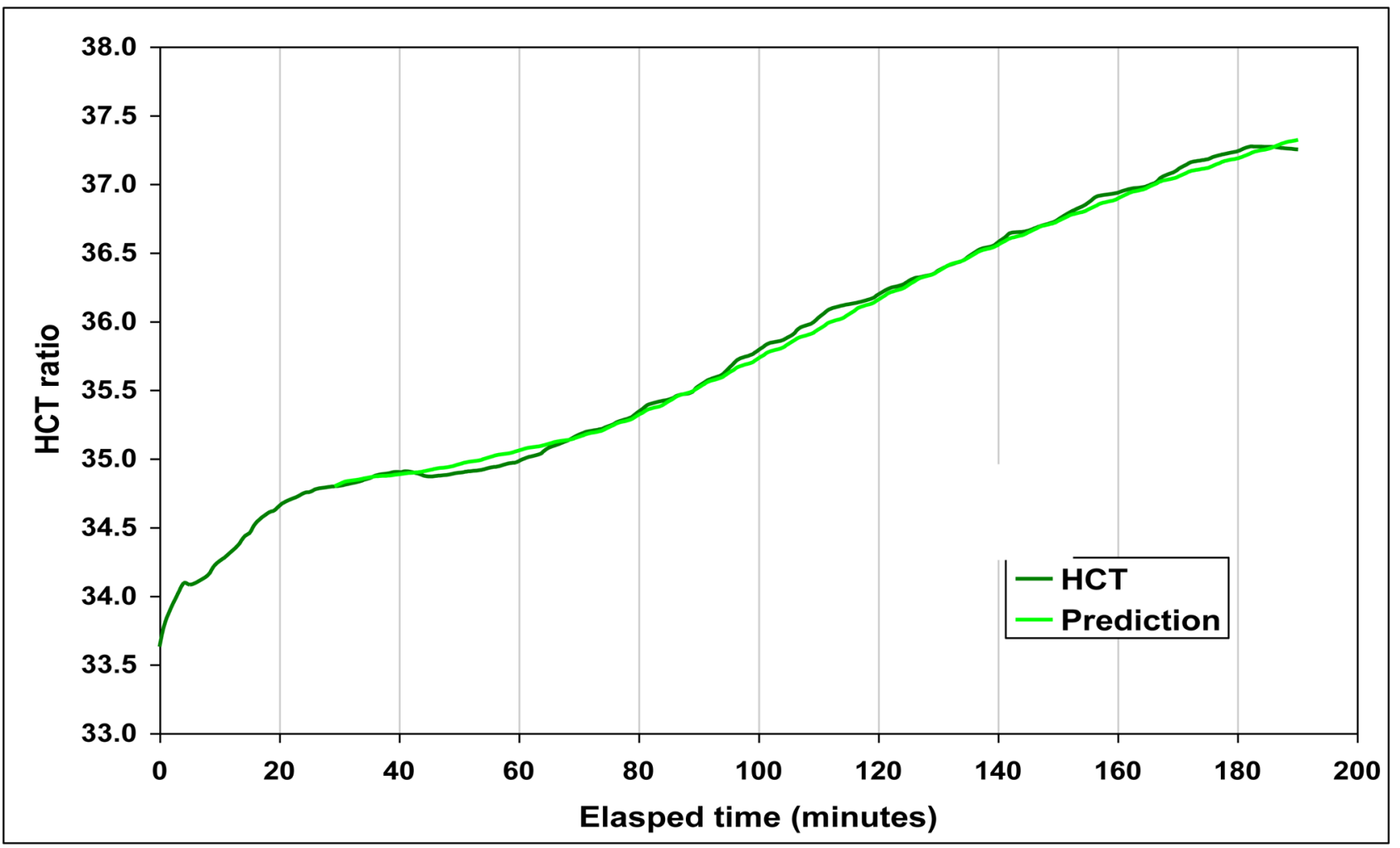

Figure 4.

Comparison of CritLine ${ }^{\circledR}$ HCT with BIS-predicted HCT 

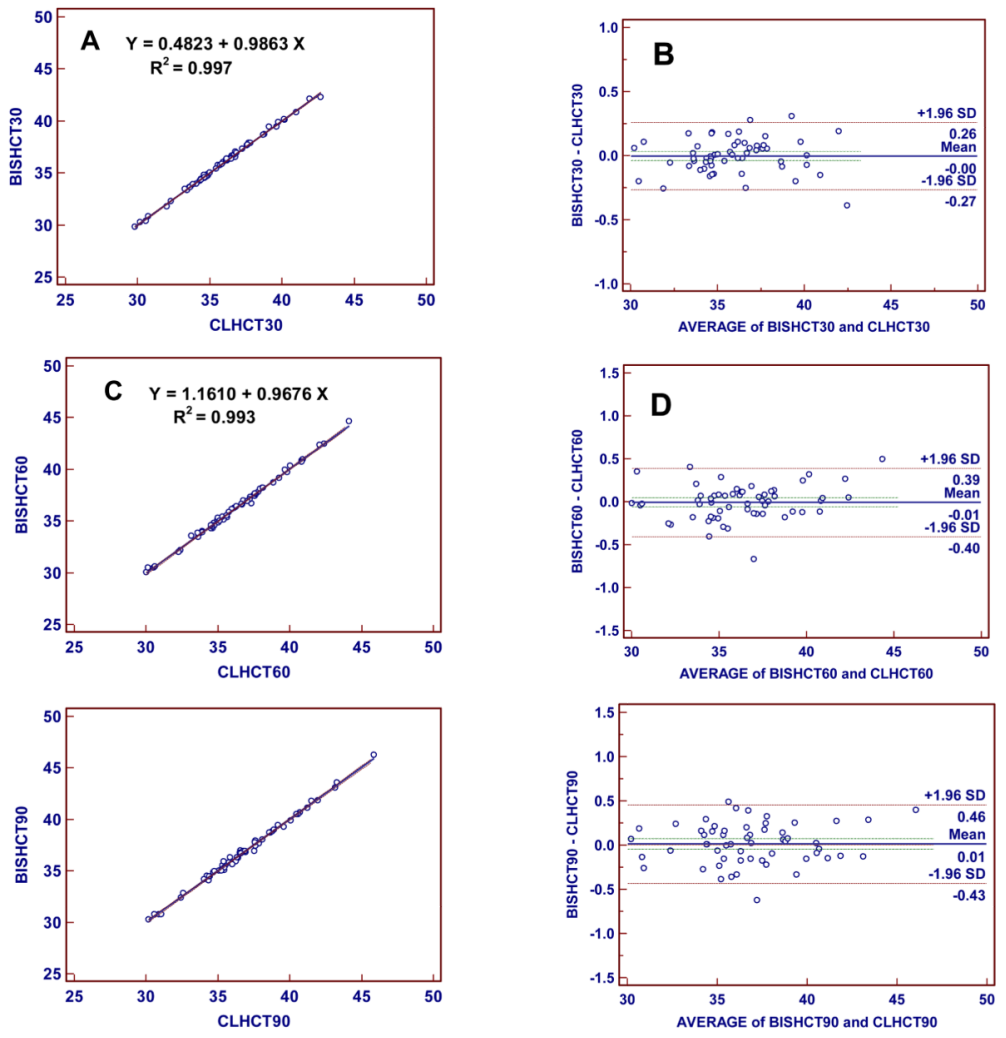

Figure 5.

Linear regression and Bland Altman analyses comparing the BISHCT and CLHCT results at specified times during dialysis 


\section{Table 3}

Differences \pm SD, limits of agreement, and independent t-test probabilities of significance between BISHCT and CLHCT values at specified elapsed times during dialysis.

\begin{tabular}{|c|c|c|c|}
\hline TIME-min. & Difference with CLHCT & Limits of agreement $(\mathbf{m e a n} \pm \mathbf{1 . 9 6 S D})$ & P (by non-pair t-test) \\
\hline 30 & $-0.00295 \pm 0.1331$ & $-0.2638 \pm 0.3180$ & 0.97 \\
\hline 60 & $-0.00864 \pm 0.2021$ & $-0.4048 \pm 0.4789$ & 0.96 \\
\hline 90 & $0.01207 \pm 0.2263$ & $-0.5338 \pm 0.5580$ & 0.95 \\
\hline 120 & $0.05661 \pm 0.2564$ & $-0.5618 \pm 0.6750$ & 0.92 \\
\hline END & $0.06066 \pm 0.2514$ & $-0.5456 \pm 0.6669$ & 0.91 \\
\hline
\end{tabular}

